\title{
Loading Rate Dependency of Dynamic Responses of Rock Joints at Low Loading Rate
}

\author{
W. Wu $\cdot$ J. C. Li $\cdot$ J. Zhao
}

Received: 16 September 2011/Accepted: 24 November 2011/Published online: 7 December 2011

(c) Springer-Verlag 2011

Keywords Split Hopkinson rock bar · Loading rate ·

Stress wave $\cdot$ Rock joints

\section{List of Symbols}

$\varepsilon_{j} \quad$ Measured strain wave at the position of the gauge $j(j=a, b)$

$\varepsilon^{p}, \varepsilon^{n}$

$\varepsilon_{j}^{p}, \varepsilon_{j}^{n}$

$\varepsilon_{i}, \varepsilon_{r}, \varepsilon_{t}$

$\varepsilon^{p-}, \varepsilon^{p+}$

$\varepsilon^{n-}, \varepsilon^{n+}$

$\varepsilon^{-}, \varepsilon^{+}$

$\dot{\varepsilon}_{f}$

$\Delta \varepsilon_{n}$

$\Delta \varepsilon_{f}$

$\Delta t$

$\sigma^{-}, \sigma^{+}$ of the gauge $j(j=a, b)$, respectively

Incident, reflected and transmitted strain waves, respectively

Positive strain waves at the front and back sides of rock joints, respectively

Negative strain waves at the front and back sides of rock joints, respectively

Strains at the front and back sides of rock joints, respectively

Strain rate of filled joints

$\Delta \varepsilon_{n} \quad$ Normalized closure of non-filled contact joints

$\Delta \varepsilon_{f} \quad$ Normalized closure of filled joints

Time interval for positive or negative strain wave propagation between the points $a$ and $b$ Stresses on the front and back sides of rock joints, respectively

W. Wu $\cdot$ J. Zhao

Ecole Polytechnique Fédérale de Lausanne (EPFL),

School of Architecture, Civil and Environmental Engineering,

Laboratory for Rock Mechanics (LMR),

1015 Lausanne, Switzerland

J. C. Li ( $\square)$

State Key Laboratory of Geomechanics and Geotechnical

Engineering, Institute of Rock and Soil Mechanics,

Chinese Academy of Sciences, Wuhan 430071, China

e-mail: jcli@whrsm.ac.cn $\sigma_{n}$

$\sigma_{f}$

$c$

E

$l$

$l_{s}$

$T$

$t_{j}^{p}$

\author{
Stress of non-filled contact joints \\ Stress of filled joints \\ Longitudinal wave propagation velocity \\ Young's modulus of the rock bar \\ Length of the rock bar \\ Thickness of the filled joint \\ Transmission coefficient \\ Time interval for positive strain wave propa- \\ gation between the points $O$ and $j(j=a, b)$ \\ Time interval for negative strain wave propa- \\ gation between the points $Y$ and $j(j=a, b)$ \\ Particle velocities at the front and back sides of \\ rock joints, respectively \\ Distance between the points $O$ and $j(j=a, b)$
}

$t_{j}^{n}$

$v^{-}, v^{+}$

$x_{j}$

\section{Introduction}

Rock discontinuities, particularly rock joints due to their universality in rock masses, play a dominant role in the behaviors and the properties of rock masses. The mechanical responses of rock joints, such as normal and shear displacements, are often induced by static and dynamic loads. Dynamic loads are commonly in the form of stress wave transmitting across rock masses. Although at a low stress level, a dynamic load may not induce rock material fracturing and fragmentation, it may however, generate significant dynamic responses of rock joints that lead to the large movement at the joint contacts and the instability of the rock masses. In addition, open joints with filling materials (e.g., sand and clay) are likely to be more sensitive due to the low strength and the high deformability of the filling materials. 
Stress wave propagation across rock joints has been investigated theoretically (e.g., Pyrak-Nolte et al. 1990; Cai and Zhao 2000; Li et al. 2010a; Zhu et al. 2011) and experimentally (e.g., Leucci and Giorgi 2006; Zhao et al. 2006, 2008; Li et al. 2010b). Many studies focus on the interaction between the stress wave and the rock joints (e.g., Li et al. 2011; Ma et al. 2011). The reflection and transmission characteristics of stress wave propagation and the dynamic response of rock joints with filling and nonfilling materials have been well understood.

The rate dependency is the another significant issue that related to the dynamic behavior of rock masses. As well known, the rock material strength increases with higher loading rate (Zhao et al. 1999; Zhao 2000; Doan and Gary 2009). However, the loading rate dependence for rock joints has received less attention, probably due to the complication of analytical solutions and the limitation of experimental methods. As the mechanical behavior of rock masses is governed by rock joints, it is necessary to evaluate the loading rate effect on rock joints.

This technical note reports a series of dynamic tests using a split Hopkinson rock bar (SHRB) on non-filled contact and filled rock joints at three low loading rates (approximately 30, 60, and $80 \mathrm{GPa} / \mathrm{s}$ ), in order to investigate the rate-dependent characteristics of the joints. The experimental results will be helpful to estimate the responses of rock masses subjected to a low loading rate impact and provide reasonable parameters for the future theoretical and numerical studies.

\section{Experimental Study}

Figure 1a shows the schematic view of the SHRB apparatus, which consists of the incident and the transmitted rock bars with the square cross section of $40 \times 40 \mathrm{~mm}$, a dynamic loading system with a striker, and a data acquisition unit.

The theory of one-dimensional wave propagation is valid for square bars, if the wavelength is much larger than the lateral dimensions of bars (Kolsky 1953). The incident and transmitted bars, made of the high-quality Dark Impala norite, are 1,500 $\mathrm{mm}$ long. The norite has high uniaxial compressive strength (average $284 \mathrm{MPa}$ ), homogeneous grain size and few visible micro cracks. The longitudinal wave velocity in the norite medium is $6,500 \mathrm{~m} / \mathrm{s}$, and the average Young's modulus is $63.6 \mathrm{GPa}$. The rock bars and the striker are carefully screened under the ultrasonic apparatus to ensure the bars have the lowest default that may influence wave propagation. This screen exercise is constantly performed.
A dynamic loading system with a compressed spring with a stiffness coefficient of $19.64 \mathrm{~N} / \mathrm{m}$ is used to instantaneously launch the striker at a low loading rate (in this study, approximately 30, 60 and $80 \mathrm{GPa} / \mathrm{s}$, respectively) to maintain elastic deformation of the bars and the striker during the impact. The norite striker has the same cross section as the bars and a length of $200 \mathrm{~mm}$.

The data acquisition unit is configurated based on the LabVIEW platform, including signal triggering, data recording and storage. Two groups of strain gauges, connected in the full bridge to average out the bending strain and to reduce the signal noise, are glued on each bar. The strain gauge stations are 200 and $400 \mathrm{~mm}$ away from the joint on each bar. The strain gauge groups are arranged at least five times side length away from the bar end to eliminate the lateral inertia effect (Vales et al. 1996; Meng and $\mathrm{Li}$ 2003), and as close to the joint as possible to minimize the influence of the material damping, in order to provide good measurements for wave separation analysis.

The non-filled contact joint is a flat sawed unpolished plane. The joint surfaces are generally considered as planar smooth (Brown 1981). In the test setup, it is assumed to be the direct contact interface between the incident bar (back end) and the transmitted bar (front end). The filled joint is simulated by inserting a sand layer in the opening at the interface of two bars. The filling material is dry quartz sands with a bulk density of $1,550 \mathrm{~kg} / \mathrm{m}^{3}$ and particle size of $1-2 \mathrm{~mm}$. To setup the test with filled joint, a gap at the interface of two bars is pre-set, filled with sands, and held together by an aluminum box with inside cross section of $41 \times 41 \times$ $20 \mathrm{~mm}$. The filling material is in a uniaxial strain state during the tests.

A rubber disc, $10 \mathrm{~mm}$ in diameter and $1 \mathrm{~mm}$ in thickness, is employed as a pulse shaper stuck at the center of the incident bar impact end, in order to generate a nondispersive low rate loading pulse, facilitate stress equilibrium in the non-filled contact joint, obtain constant strain rate deformation in both joints and protect the contacting ends of the striker and the incident bar.

A wave separation technique is adopted for the data analysis. The recorded signal is always superposed by two waves in the opposite directions because of the short length of rock bars. The wave along the loading direction is defined to be the positive wave, while that opposite to the loading direction is the negative wave, indicating the superscripts " $p$ " and " $n$ ", respectively. When an incident wave propagates from the front end $O$ of the bar, it is reflected at the back end $Y$, as illustrated in Fig. 1b. Assuming that the recordings from the two gauge groups are $\varepsilon_{a}(t)$ and $\varepsilon_{b}(t)$, which are superposed by the opposite waves, $\varepsilon_{a}^{p}(t)$ and $\varepsilon_{a}^{n}(t)$ for the 
Fig. 1 The split Hopkinson rock bar, a schematic view, b characteristic diagram in a rock bar (a)
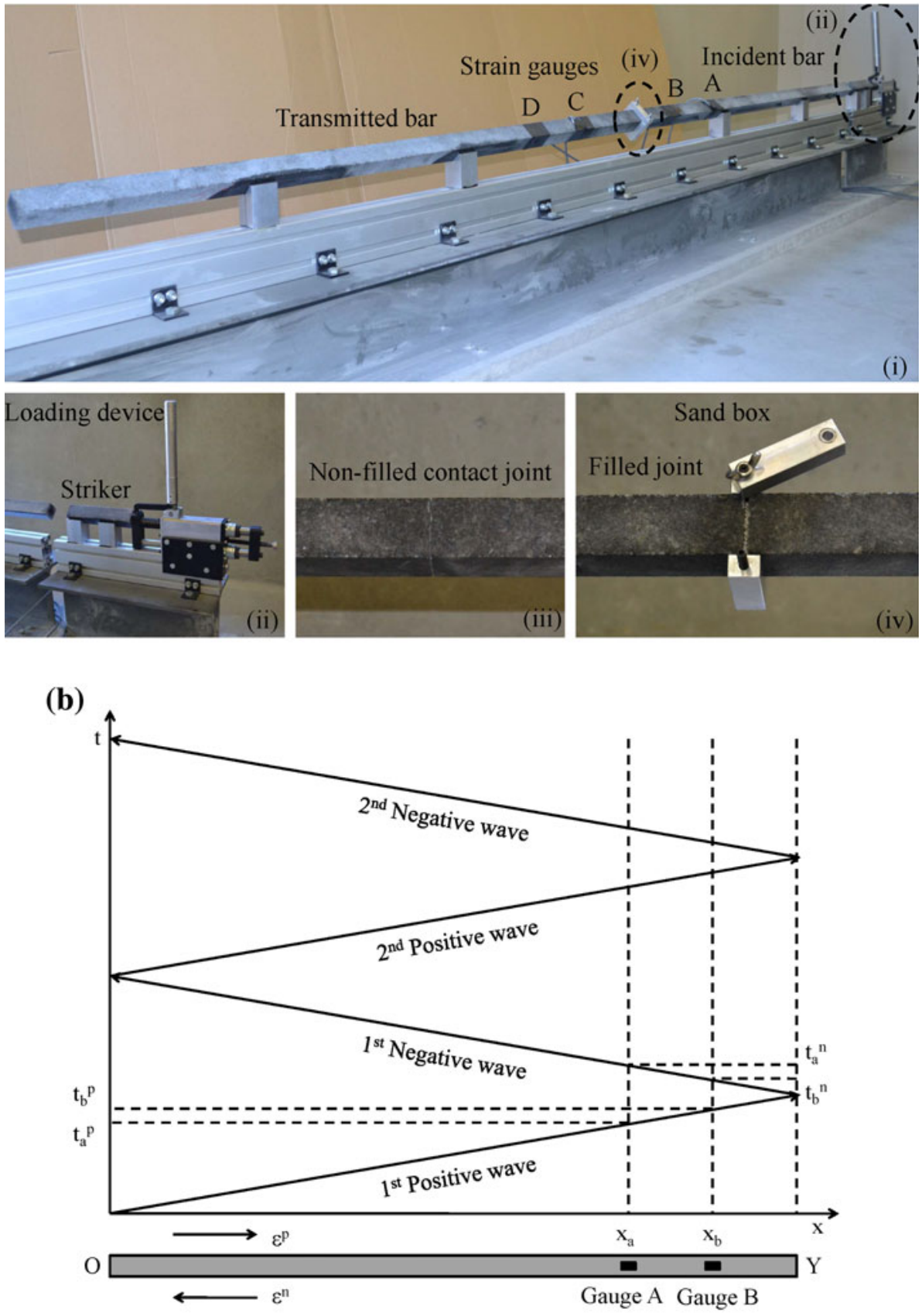

group A, and $\varepsilon_{b}^{p}(t)$ and $\varepsilon_{b}^{n}(t)$ for the group B. Defining $t_{a}^{p}=x_{a} / c, \quad t_{b}^{p}=x_{b} / c, t_{a}^{n}=\left(l-x_{a}\right) / c, t_{b}^{n}=\left(l-x_{b}\right) / c$, and $\Delta t=t_{b}^{p}-t_{a}^{p}=t_{b}^{n}-t_{a}^{n}=\left(x_{b}-x_{a}\right) / c, l$ is the bar length, $x_{a}$ and $x_{b}$ are the distances from the group $\mathrm{A}$ and the group $\mathrm{B}$ to the front end, and $c$ is the longitudinal wave velocity. The iterative algorithm (Zhao and Gary 1997; Meng and $\mathrm{Li} 2003$ ) to separate the strain time responses $\varepsilon_{a}(t)$ and $\varepsilon_{b}(t)$ is,
$\left\{\begin{array}{l}\varepsilon_{a}^{n}(t)=\varepsilon_{b}^{n}(t-\Delta t) \\ \varepsilon_{a}^{p}(t)=\varepsilon_{a}(t)-\varepsilon_{a}^{n}(t)=\varepsilon_{a}(t)-\varepsilon_{b}^{n}(t-\Delta t) \\ \varepsilon_{b}^{p}(t)=\varepsilon_{a}^{p}(t-\Delta t) \\ \varepsilon_{b}^{n}(t)=\varepsilon_{b}(t)-\varepsilon_{b}^{p}(t)=\varepsilon_{b}(t)-\varepsilon_{a}^{p}(t-\Delta t)\end{array}\right.$

It shall be noted that the strain values are zero until the arrival of the positive or negative wave. 
For any point on the bar, the positive and negative waves can be derived by time shifting the positive wave at the group $\mathrm{A}$ and the negative wave at the group B, which is

$\varepsilon^{p}(t)=\varepsilon_{a}^{p}\left(t-\frac{l-x_{a}}{c}\right), \quad \varepsilon^{n}(t)=\varepsilon_{b}^{n}\left(t+\frac{l-x_{b}}{c}\right)$

The stress time responses of the joint sides can be obtained as,

$\left\{\begin{array}{l}\sigma^{-}(t)=E \varepsilon^{-}(t)=E\left(\varepsilon^{p-}(t)+\varepsilon^{n-}(t)\right) \\ \sigma^{+}(t)=E \varepsilon^{+}(t)=E\left(\varepsilon^{p+}(t)+\varepsilon^{p+}(t)\right)\end{array}\right.$

where $\varepsilon^{p-}(t)$ and $\varepsilon^{n-}(t), \varepsilon^{p+}(t)$ and $\varepsilon^{n+}(t)$ are the opposite waves at the back end of the incident bar and the front end of the transmitted bar, respectively. The symbols "-" and "+" denote the back end of the incident bar (the front side of the joint) and the front end of the transmitted bar (the back side of the joint), respectively.

For the non-filled contact joint, as stress equilibrium on the joint, the stress time response $\sigma_{n}(t)$ is the average of stresses at the joint sides,

$\sigma_{n}(t)=\frac{\sigma^{-}(t)+\sigma^{+}(t)}{2}$

The strain time response cannot be determined by the principal of split Hopkinson pressure bar test, as it is difficult to measure the real joint thickness, therefore, the strain time responses of the joint sides are calculated separately,

$\varepsilon^{-}(t)=\frac{v^{-}(t)}{c}=\varepsilon^{p-}(t)-\varepsilon^{n-}(t)$,

$\varepsilon^{+}(t)=\frac{v^{+}(t)}{c}=\varepsilon^{p+}(t)-\varepsilon^{n+}(t)$

The normalized closure time response $\Delta \varepsilon_{n}(t)$ is determined by the joint closure over the original length, and equal to the sum of the strains at two sides,

$\Delta \varepsilon_{n}(t)=\varepsilon^{-}(t)+\varepsilon^{+}(t)$

For the filled joint, the stress time response in the filled joint is non-uniformity. The stress time response $\sigma_{f}(t)$ at the front end of the transmitted bar represents the dynamic response of the joint, indicating the energy portion that can travel through the densifying sand layer. $l_{s}$ is the thickness of the filled joint. According to the strain rate time response of the sand layer,

$$
\begin{aligned}
\dot{\varepsilon}_{f}(t) & =\frac{v^{-}(t)-v^{+}(t)}{l_{s}} \\
& =\frac{c}{l_{s}}\left[\left(\varepsilon^{p-}(t)-\varepsilon^{n-}(t)\right)-\left(\varepsilon^{p+}(t)-\varepsilon^{n+}(t)\right)\right]
\end{aligned}
$$

the normalized closure time response $\Delta \varepsilon_{f}(t)$ is,

$$
\begin{aligned}
\Delta \varepsilon_{f}(t) & =\int_{0}^{t} \dot{\varepsilon}_{f}(t) d t \\
& =\frac{c}{l_{s}} \int_{0}^{t}\left[\left(\varepsilon^{p-}(t)-\varepsilon^{n-}(t)\right)-\left(\varepsilon^{p+}(t)-\varepsilon^{n+}(t)\right)\right] d t
\end{aligned}
$$

The loading rate can be determined by the slope of the pre-peak linear portion of the stress time response at the front side of the joint. Similarly, the joint stiffness is estimated as the slope of the pre-peak linear portion of the stress versus normalized closure response.

The transmission coefficient is defined as,

$$
T=\frac{\max \left(\varepsilon_{t}(t)\right)}{\max \left(\varepsilon_{i}(t)\right)}=\frac{\max \left(\varepsilon_{t}(t)\right)}{-\min \left(\varepsilon_{r}(t)\right)+\max \left(\varepsilon_{t}(t)\right)}
$$

where $\varepsilon_{i}(t), \varepsilon_{r}(t)$, and $\varepsilon_{t}(t)$ are the time responses of the incident, reflected and transmitted waves, respectively.

\section{Experimental Results and Discussion}

A total of 60 dynamic tests were conducted on the nonfilled contact and filled joints at three low loading rates (approximately 30, 60, and $80 \mathrm{GPa} / \mathrm{s}$ ). Figure 2 plots the stresses versus normalized closure responses of the joints. The stress and normalized closure of the non-filled contact joint continuously increase with the increase of loading rate. The filled joint responds with a similar trend, nevertheless, for a given loading rate the peak value of the closure decreases with increasing thickness.

Figure 3 reveals that the maximum stress on both joints increases with increasing loading rate, similar to the loading rate effect on rock material strength. The maximum stress of the non-filled contact joint has a rapid rise over the loading rates. Nonetheless, the existence of the sand layer significantly dissipates the impact energy resulting in a low stress increment. The larger the thickness is, the more energy is absorbed, and the lower stress is achieved.

For the loading rates of $30-80 \mathrm{GPa} / \mathrm{s}$ in the study, the stiffness of the non-filled contact joint nearly keeps constant, while the stiffness of the filled joint displays loading rate dependency (Fig. 4). The impact in the present loading rate range keeps the non-filled contact joint in the elastic deformation range. The deformation of the filled joint with three thicknesses has limited difference at the loading rate of $80 \mathrm{GPa} / \mathrm{s}$, while with lower loading rate, larger thickness shows a significant reduction of stiffness owing to the high deformability of the uncompacted sand layer. The stiffness of the non-filled contact joint is much higher than that of the filled joint, which means the existence of the joint 

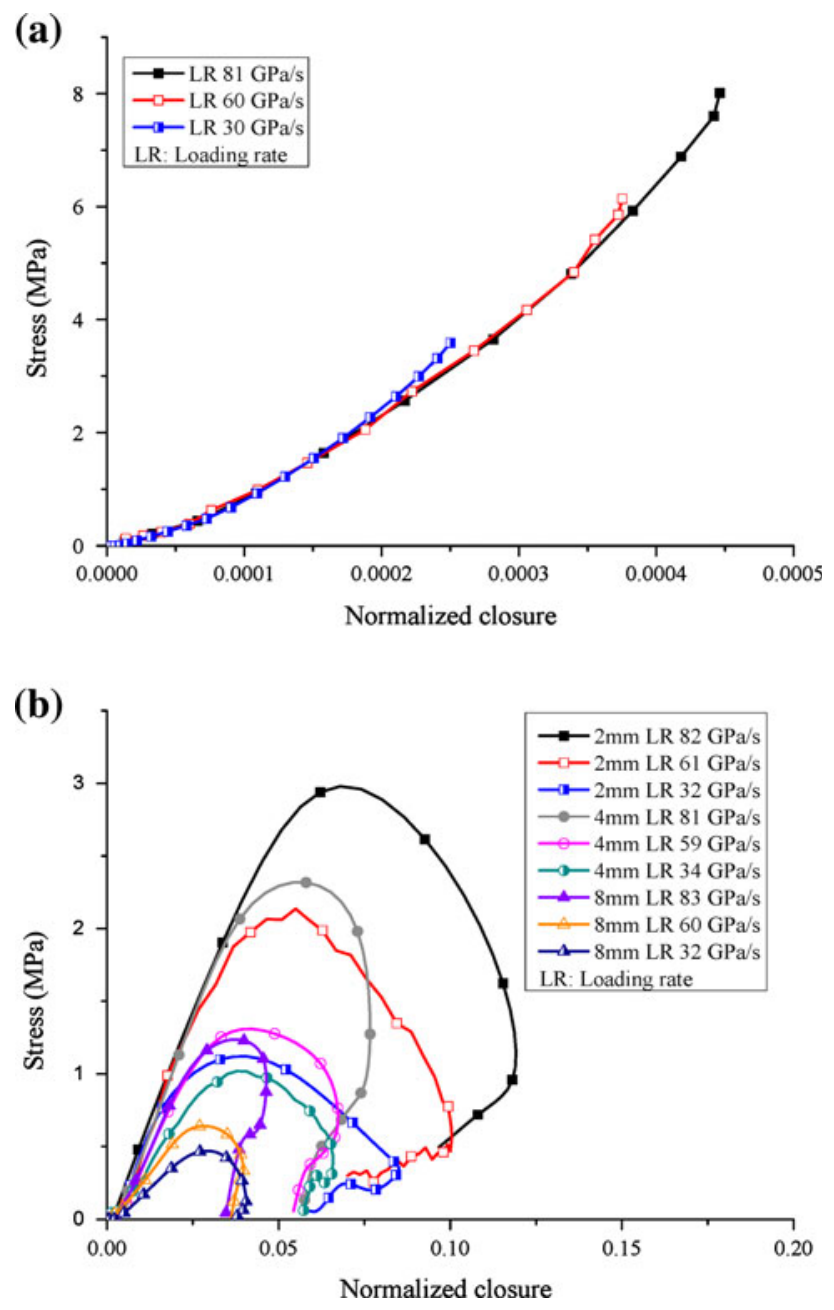

Fig. 2 Dynamic responses of rock joints, a non-filled contact joint, b filled joint

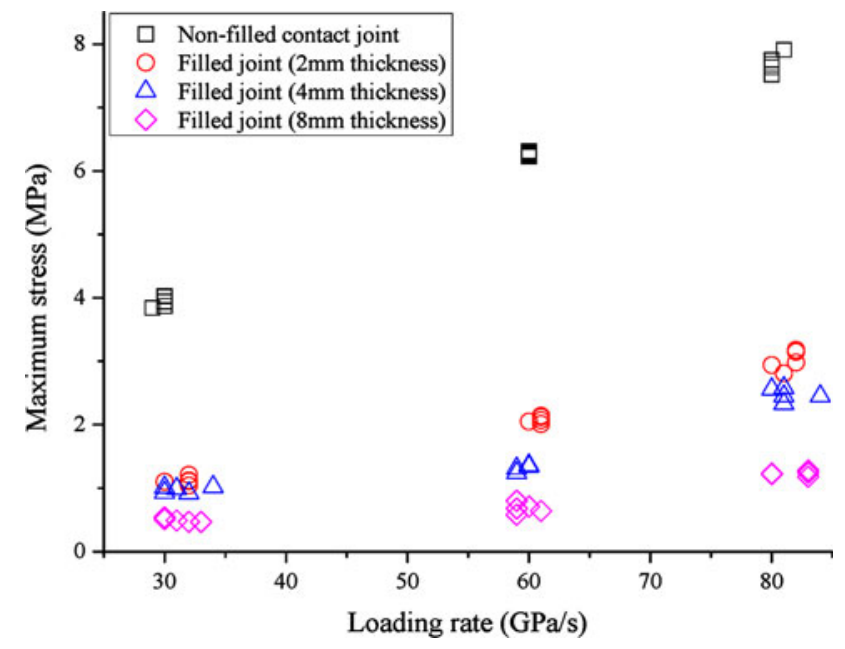

Fig. 3 The variation of maximum stress with the loading rate

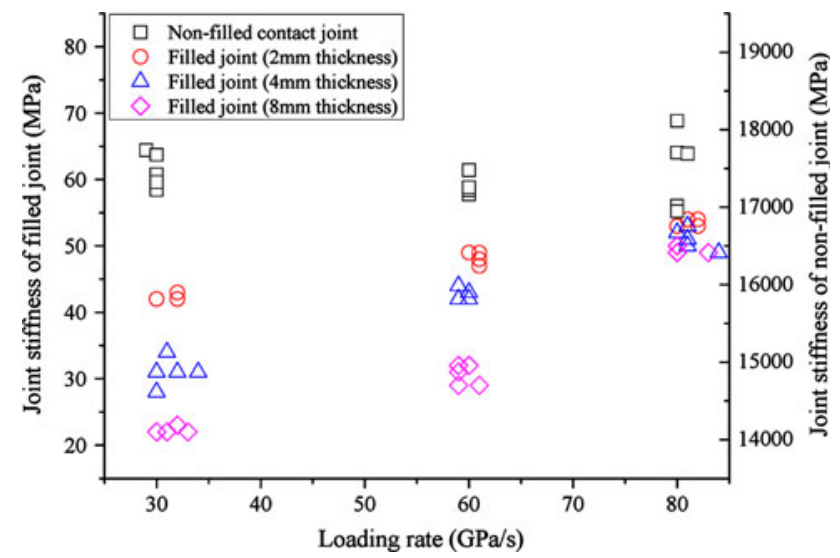

Fig. 4 The variation of joint stiffness with the loading rate

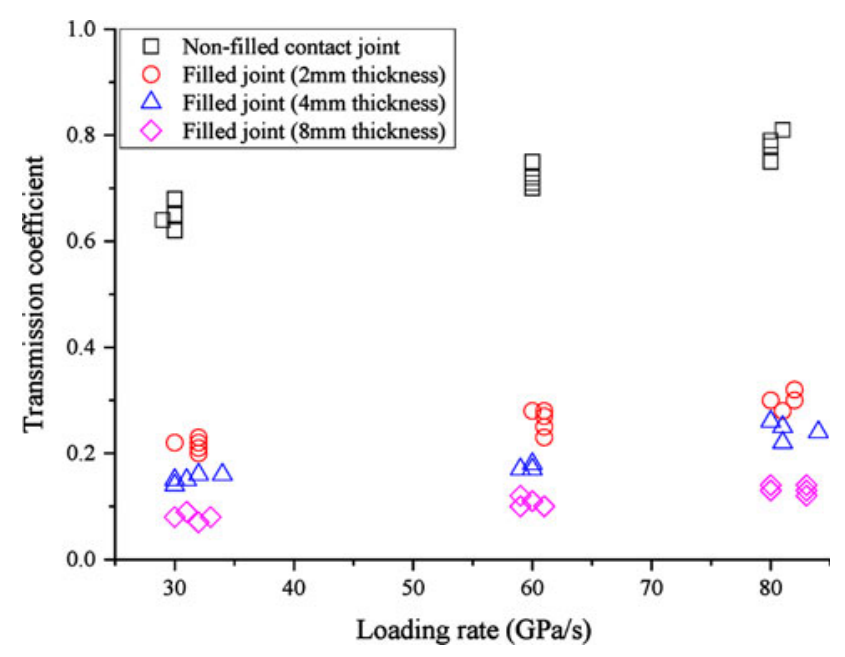

Fig. 5 The variation of transmission coefficient with the loading rate

aperture and the filling materials makes rock mass more deformable.

Figure 5 demonstrates that the transmission coefficient $T$ for the non-filled contact joint clearly increases with increasing loading rate. For the filled joint, the values are scattered with different joint thickness and loading rates, but it can still be observed from the figure that the coefficient increases slightly with the increase of loading rate. The transmission coefficient for the filled joint is much lower than that of the non-filled contact joint. Wave attenuates much highly due to the joint aperture and the low stiffness filling material.

For the non-filled contact joint, the increase of dynamic loading results in the contact of the joint interface gradually closer and a better contact and seismic coupling (e.g., Zhao et al. 2006). The joint stiffness and transmission coefficient then become higher. For the filled joint, the dynamic loading initially compresses the sand layer, rearranges the particle contact, even crushes the weak particles, but cannot totally close the air voids. The densification 
process continues during wave propagation causing stress non-equilibrium. The stress equilibrium maybe depends on the sand densification process and particle size distribution, which can be obtained theoretically without the air-filled porosity (Felice et al. 1987). It is also evident that a higher loading rate leads to a larger deformation (closure) of the joint and a higher transmitted energy across the joint (Fig. 2b). This also implies that higher joint stiffness and transmission coefficient is caused by higher loading rate.

\section{Conclusions}

The split Hopkinson rock bar tests on the non-filled contact and filled rock joints were conducted at three low loading rates (approximately 30, 60, and $80 \mathrm{GPa} / \mathrm{s}$ ). The results indicate that the non-filled contact joint displays stress equilibrium, while the filled joint exhibits stress discontinuity. The maximum stresses of both joints increase with increasing loading rate, similar to the loading rate effect on rock material strengths. The stiffnesses of both joints also increase with the higher loading rate, but for the filled joint it decreases remarkably with increasing thickness at fixed loading rates. It is also found that the filled joint displays lower strength and larger deformation than the non-filled contact joint, which likely induces the instability of rock masses or underground structures.

The transmission coefficient for the filled joint has less increment than that for the non-filled contact joint with increasing loading rate. It is also observed that wave attenuates much highly due to the joint aperture and the low stiffness filling material.

The SHRB test is an excellent experimental technique to study the interaction between the stress waves and rock joints, by characterizing the joint stiffness and the transmission coefficient, and the rate-dependent behaviors of rock joints. However, the loading rate may have to be limited to about $100 \mathrm{GPa} / \mathrm{s}$, due to the low tensile strength of rock material.

Acknowledgments This work is sponsored by the Swiss National Science Foundation (200021_124846). The equipment is partially supported by Swiss National Science Foundation (200021_116536). The authors also would like to thank Mr. J.-F. Mathier, Mr. L. Gastaldo and Mr. L.F. Morier of EPFL-LMR, for their assistance in equipment setup and test preparation.

\section{References}

Brown ET (1981) Rock characterization testing and monitoring: ISRM suggested methods. Pergamon, Oxford

Cai JG, Zhao J (2000) Effects of multiple parallel fractures on apparent attenuation of stress waves in rock masses. Int J Rock Mech Min Sci 37:661-682

Doan ML, Gary G (2009) Rock pulverization at high strain rate near the San Andreas fault. Nature Geosci 2:709-712

Felice CW, Gaffney ES, Brown JA, Olsen JM (1987) Dynamic high stress experiments on soil. Geotech Test J 10:192-202

Kolsky H (1953) Stress waves in solids. Oxford University Press, London

Leucci G, Giorgi LD (2006) Experimental studies on the effects of fracture on the $\mathrm{P}$ and $\mathrm{S}$ wave velocity propagation in sedimentary rock ("Calcarenite del Salento"). Eng Geol 84:130-142

Li JC, Ma GW, Zhao J (2010a) An equivalent viscoelastic model for rock mass with parallel joints. J Geophys Res 115:B00305

Li JC, Ma GW, Huang X (2010b) Analysis of wave propagation through a filled rock joint. Rock Mech Rock Eng 43:789-798

Li JC, Ma GW, Zhao J (2011) Analysis of stochastic seismic wave interaction with a slippery rock joint. Rock Mech Rock Eng 44:85-92

Ma GW, Li JC, Zhao J (2011) Three-phase medium model for filled rock joint and interaction with stress waves. Int J Numer Anal Geomech 35:97-110

Meng H, Li QM (2003) An SHPB set-up with reduced time-shift and pressure bar length. Int J Impact Eng 28:677-696

Pyrak-Nolte LJ, Myer LR, Cook NGW (1990) Transmission of seismic waves across single natural fractures. J Geophys Res 95:8617-8638

Vales F, Moravka S, Brepta R, Cerv J (1996) Wave propagation in a thick cylindrical bar due to longitudinal impact. JSME Int J Ser A 39:60-70

Zhao J (2000) Applicability of Mohr-Coulomb and Hoek-Brown strength criteria to dynamic strength of brittle rock materials. Int J Rock Mech Min Sci 37:1115-1121

Zhao H, Gary G (1997) A new method for the separation of waves: application to the SHPB technique for an unlimited duration of measurement. J Mech Phys Solid 45:1185-1202

Zhao J, Li HB, Wu MB, Li TJ (1999) Dynamic uniaxial compression tests on a granite. Int J Rock Mech Min Sci 36:273-277

Zhao J, Cai JG, Zhao XB, Li HB (2006) Experimental study of ultrasonic wave attenuation across parallel fractures. Geomech Geoeng An Int J 1:87-103

Zhao J, Cai JG, Zhao XB, Li HB (2008) Dynamic model of fracture normal behavior and application to prediction of stress wave attenuation across fractures. Rock Mech Min Sci 41:671-693

Zhu JB, Perino A, Zhao GF, Barla G, Li JC, Ma GW, Zhao J (2011) Seismic response of a single and a set of filled joints of viscoelastic deformational behavior. Geophys J Int 186:1315-1330 